\title{
3. OBSERVATIONS OF COSMIC X-RAY SOURCES BY THE MIT INSTRUMENT ON THE OSO-7*
}

\author{
G. W. CLAR K \\ Dept. of Physics and Center for Space Research, Massachusetts Institute of \\ Technology, Cambridge, Mass., U.S.A.
}

\begin{abstract}
The Seventh Orbiting Solar Observatory, OSO-7, carries on board a 1-60 keV X-ray detector for the purpose of measuring the positions, spectra and time variations of X-ray sources. The instrument is described herein, and several topics under investigation and results in publication are mentioned.
\end{abstract}

A $1-60 \mathrm{keV} \mathrm{X}$-ray detector ** has been operating successfully in the wheel section of the Seventh Orbiting Solar Observatory since it was launched on September 29, 1971. The instrument was prepared at MIT for the purposes of measuring the positions, spectra and time variation of X-ray sources.

The detectors are two banks of gas proportional counters behind tubular collimators that define two circular fields of view, one with a $1^{\circ} \mathrm{FWHM}$ centered $15^{\circ}$ above the wheel plane, and the other with a $3^{\circ} \mathrm{FWHM}$ centered $15^{\circ}$ below the wheel plane. Figure 1 shows the arrangement of the detectors and collimators and their energy response functions. As the wheel rotates with a period of about $2 \mathrm{~s}$ the fields of view sweep out two circular scan bands in the sky. The counts are accumulated in 256 azimuth bins, the contents of which are telemetered sequentially with a period of $191 \mathrm{~s}$. As the spin axis is precessed to maintain it at an angle near $90^{\circ}$ from the Sun direction, the scan bands gradually sweep over the sky. Figure 2 is a summary of the sky exposure which was obtained during the first six months in orbit.

More complete descriptions of the instrument and its performance characteristics have been submitted for publication (Clark et al., 1972a). Results have been obtained so far on the following topics:

(1) Measurement of the position and spectrum of Hercules X-1 ${ }^{* * *}$ (see Figures 3 and 4) (Clark et al., 1972b).

(2) 20-day observation of Scorpio X-1 with simultaneous radio observations (see Figure 5).

(3) The spectrum and time variations of a transient X-ray source in Lupus.

(4) Evidence of the interstellar absorption of $1-3 \mathrm{keV}$ background radiation at the galactic equator near $l^{\mathrm{II}}=145^{\circ}$.

(5) Upper limits on the X-ray luminosity of three extragalactic supernovae near the times of their optical discovery.

* Supported in part by Contract NGL 22-009-115 from the National Aeronautics and Space Administration.

** Participating scientists at MIT are H. V. Bradt, C. Cañizares, D. Hearn W. H. G. Lewin, T. H. Markert, H. W. Schnopper, and G. Sprott.

*** Note added in proof: Her X-1 has been identified with the variable $\mathrm{HZ}$ Herculis which lies within $0.2^{\circ}$ of the center of the OSO-7 error circle (Liller, 1972). 

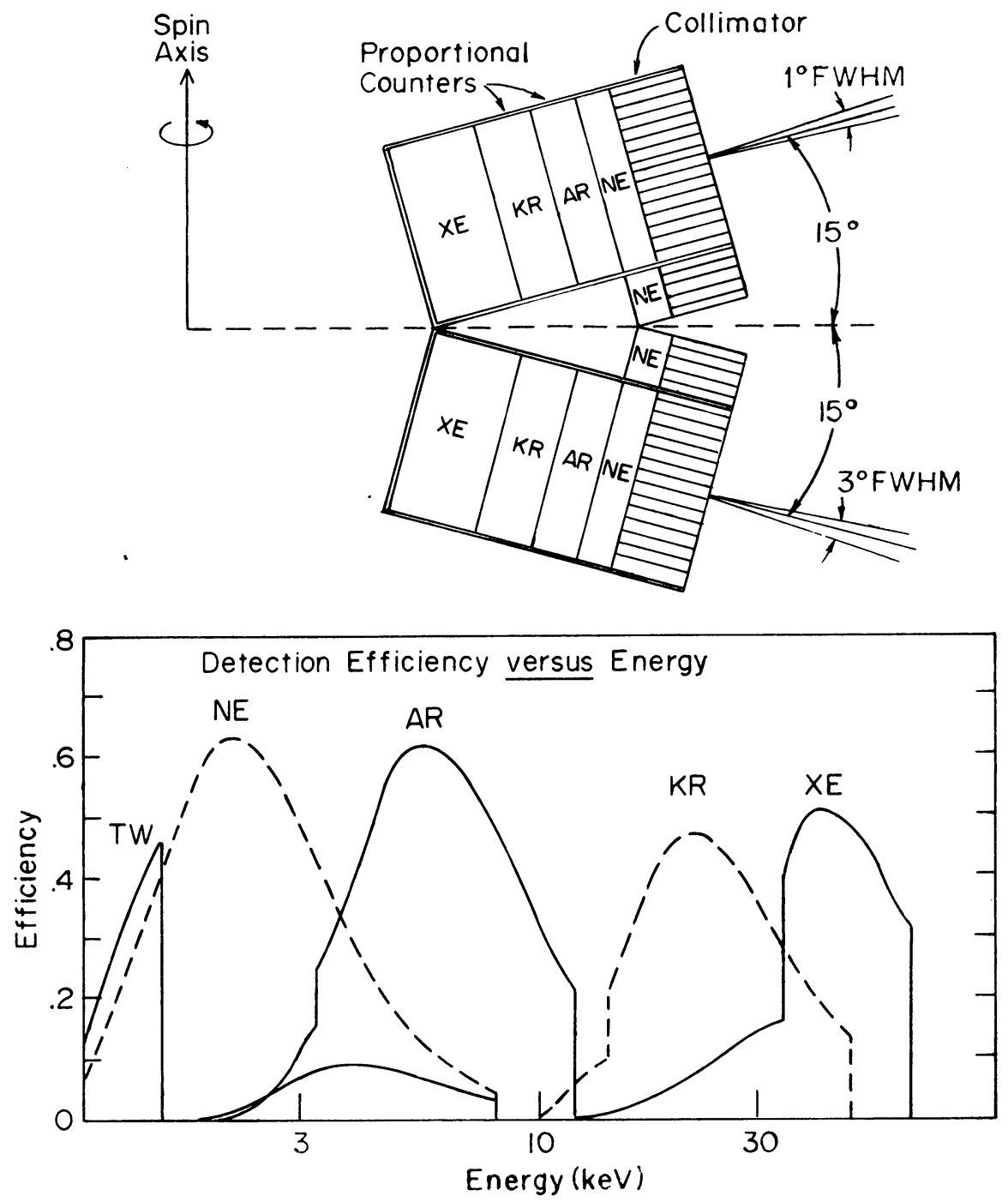

Fig. 1. Schematic diagram of the 1-60 keV X-ray detectors on the OSO-7 and plots of the detection efficiencies of the proportional counters. 


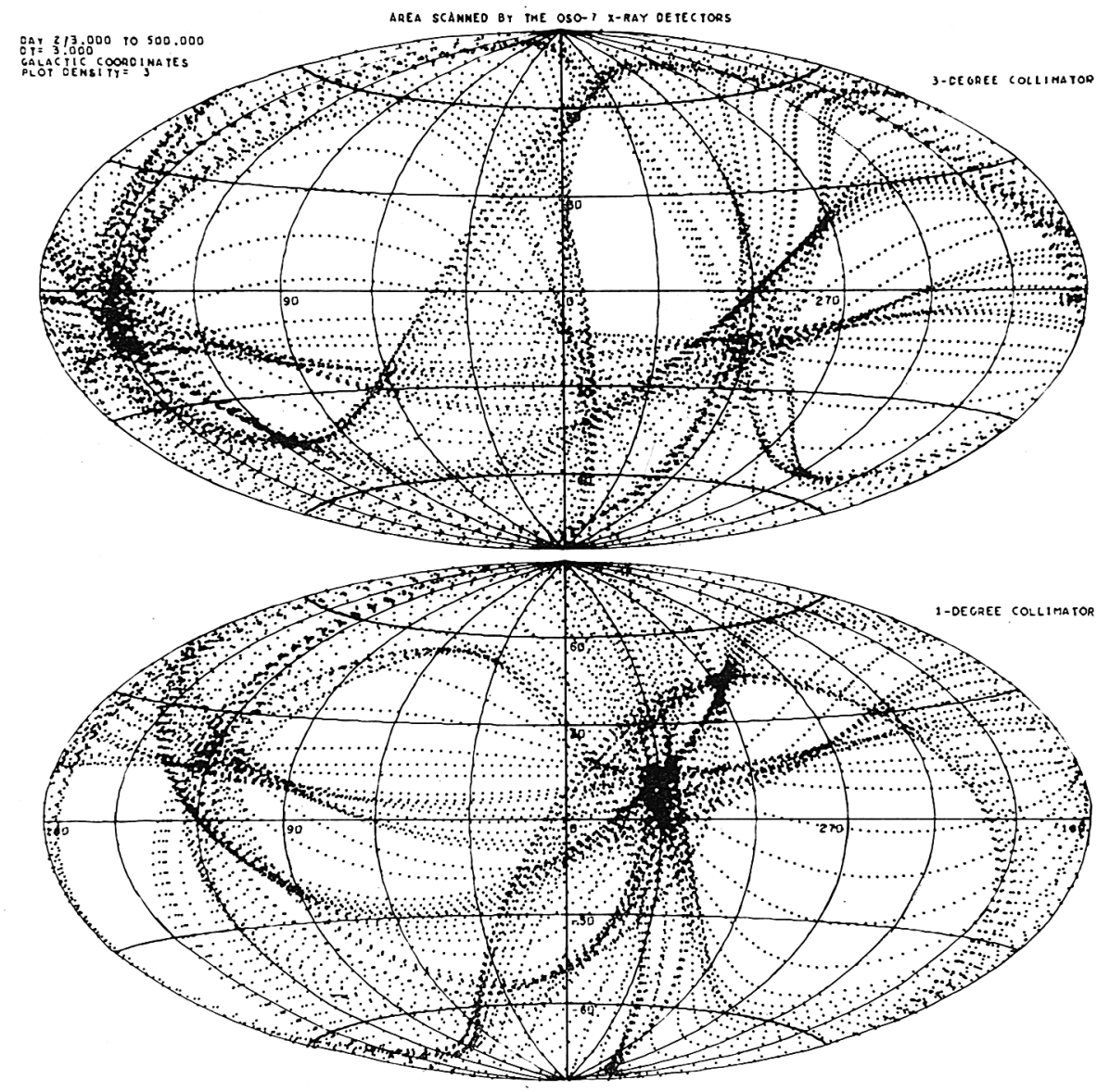

Fig. 2. Maps of sky exposure in galactic coordinates during the first six months of observations. 


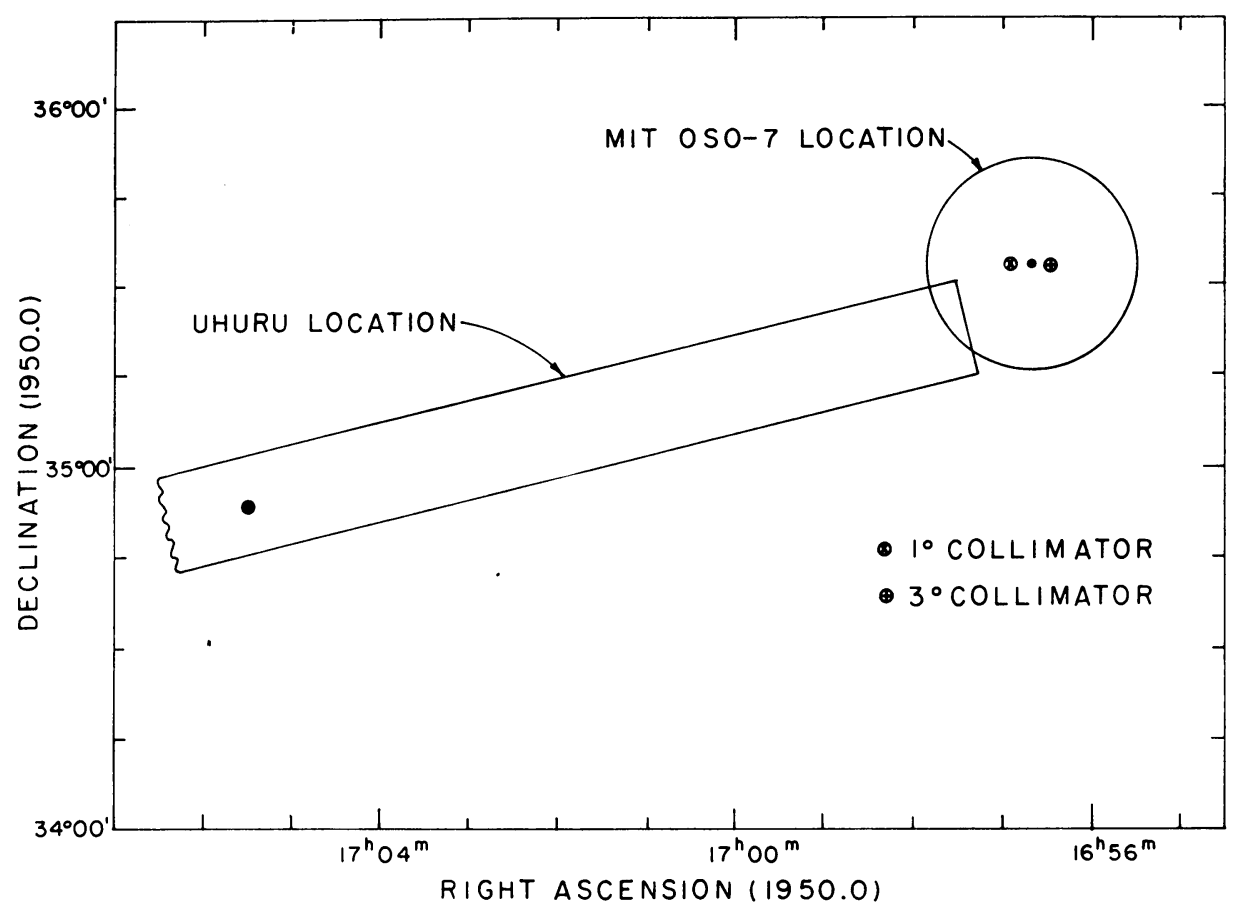

Fig. 3. Estimated position of Hercules $\mathrm{X}-1$. 


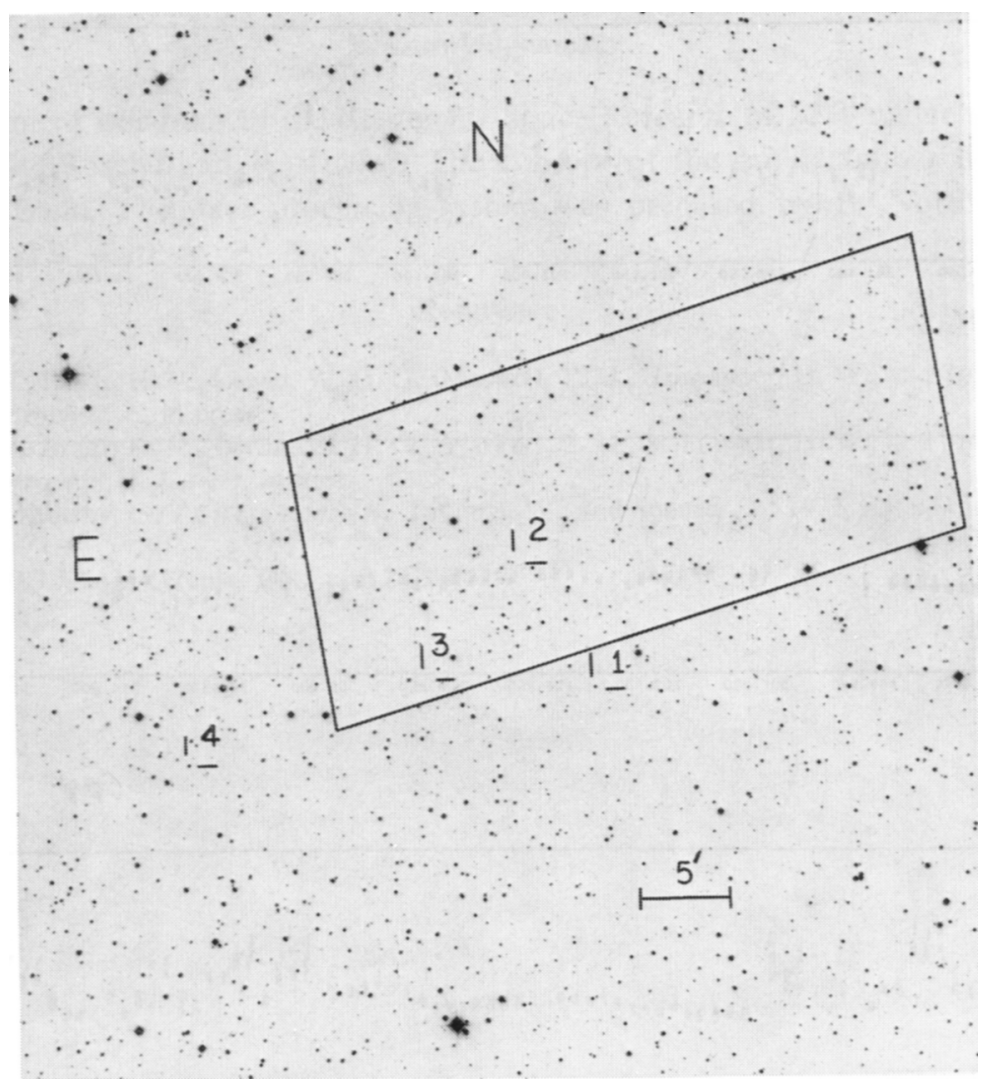

Fig. 4. Enlargement of a Palomar Sky Survey print (National Geographic Society) of the Her X-1 region. The parallelogram represents the approximate intersection of the OSO-7 error box and the extended UHURU error box. Four radio sources $(2695 \mathrm{MHz})$ are indicated (from Doxsey et al., 1972). [Editor's note in proof: The recently proposed and well substantiated optical candidate, HZ Herculis, (Davidsen et al. and Forman et al., in press) is the moderately bright star $4.0 \mathrm{~mm}$ north and $19.8 \mathrm{~mm}$ east of the southwest corner of the parallelogram. It is the end of the handle of a 'dipper' composed of fainter stars.] 

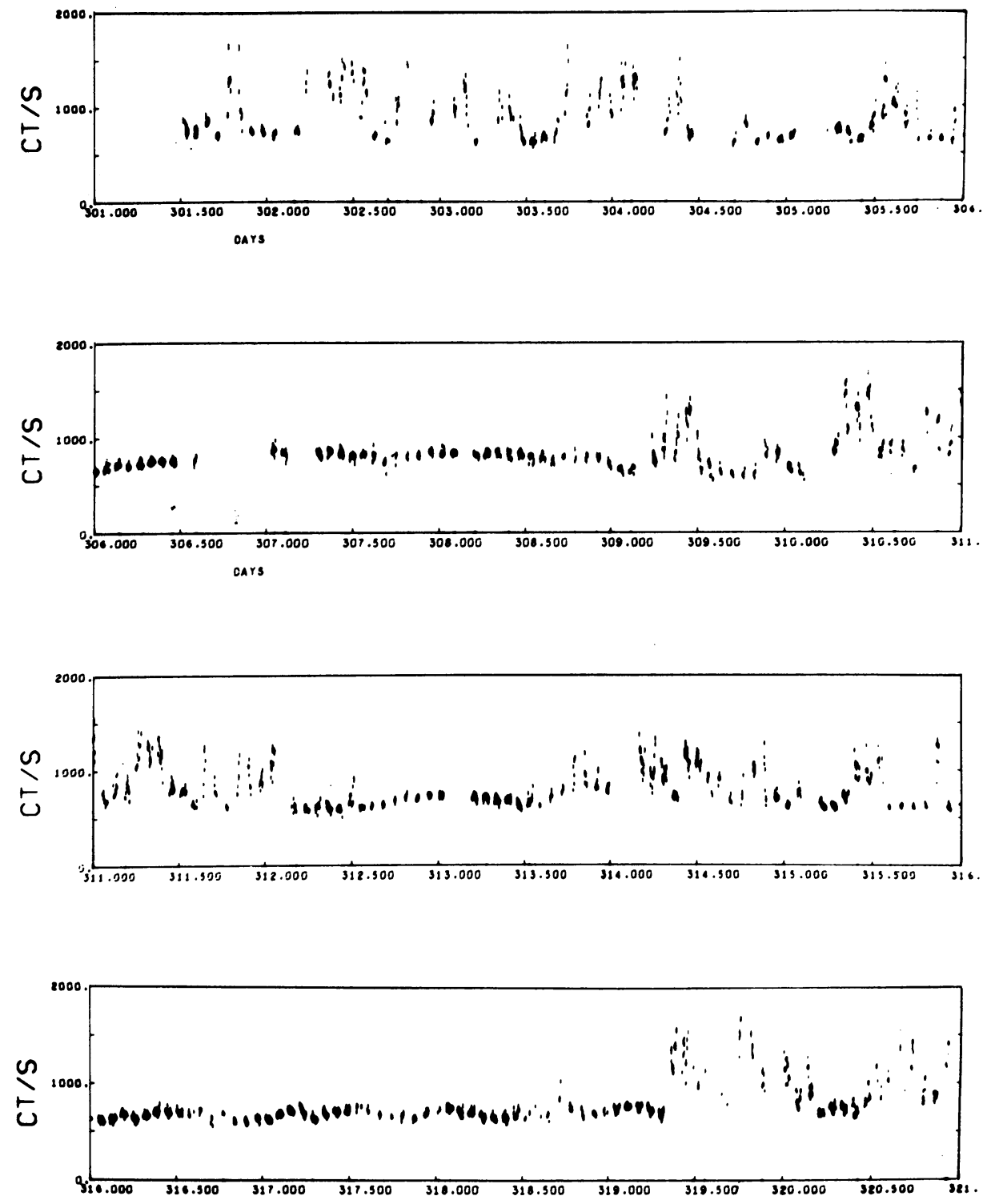

Fig. 5. Twenty-day observation of Sco X-1 as recorded in the $3^{\circ}$ AR detector. 


\section{Acknowledgements}

The instrument was built at the Center for Space Research of MIT under the supervision of R. Rasche and R. Taylor. The concept of the multichamber detector is due to J. Stein. The data processing system was prepared by P. Northridge, T. Thorsos and S. Watt.

\section{Reference}

Clark, G. W., Bradt, H. V., Lewin, W. H. G., Markert, T. H., Schnopper, H. W., and Sprott, G. F.: 1972a, Astrophys. J., in press.

Clark, G. W., Bradt, H. V., Lewin, W. H. G., Markert, T. H., Schnopper, H. W., and Sprott, G. F.: 1972b, Astrophys. J. Letters, in press.

Doxsey, R., Murthy, G. T., Rappaport, S., Zaumen, W., and Spencer, J.: 1972, Astrophys. J. Letters 176, L15.

Liller, W.: 1972, IAU Circular 2415 and 2427. 